\title{
Renforcement des sols alluvionnaires par injection de coulis de ciment ultrafin
}

\section{DANO}

P.-Y. HICHER

Laboratoire de génie civil de Nantes Saint-Nazaire

École centrale de Nantes BP 92101

44321 Nantes

Christophe.Dano@ ec-nantes.fr

Pierre-Yves.Hicher@ ec-nantes.fr

M. VARJABEDIAN

Régie autonome des transports parisiens

Bâtiment Le Périastre LAC P61, VdF2 40 bis, rue Roger Salengro 94724 Fontenay-sous-Bois Michele.Varjabedian@ratp.fr

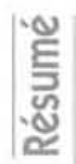

L'injection d'un coulis de ciment ultrafin dans un terrain alluvionnaire constitue un traitement de terrain efficace qui permet de construire un ouvrage souterrain sans porter préjudice à l'intégrité structurelle du bâti existant. Afin de prendre en compte, d'une manière rationnelle, l'amélioration des propriétés mécaniques des sols apportée par l'injection, on a conduit une campagne d'essais triaxiaux sur des échantillons vierges de tout traitement puis sur des échantillons injectés en laboratoire. On montre comment I'injection de coulis influe sur le comportement mécanique des sols. On propose enfin des facteurs d'amélioration des paramètres du modèle élastique plastique parfait et la façon dont ils peuvent être intégrés dans les calculs d'ouvrages.

Mots-clés : injection, coulis, alluvions, renforcement, essai triaxial.

\section{Improvement of alluvial soils by microfine cement grout injection}

The injection of a microfine cement grout into alluvial deposits is an effective soil treatment in order to construct a tunnel without damage for the surrounding structures. Triaxial tests on uncemented soils and on grouted soils injected in the laboratory are carried out in order to rationally take into account the improvement of mechanical properties of soils induced by the injection. We also show the differences in the mechanical behaviour of soils due to the injection. Finaliy, we propose improvement ratios of the parameters of the elastic plastic model and the way to integrate them into structural design.

Key words : injection, grout, alluvial deposits, reinforcement, triaxial tests. 


\section{Introduction}

En région parisienne ou dans d'autres grandes agglomérations à travers le monde, la Régie autonome des transports parisiens (RATP) est impliquée dans des programmes de construction d'infrastructures de transport. Un grand nombre de ces infrastructures (stations, tunnels) et de leurs ouvrages annexes (couloirs de correspondance, puits de ventilation, poste de redressement, ouvrages d'épuisement des eaux...) est implanté en souterrain, à des profondeurs comprises entre 0 et 30 mètres. Les contraintes de conception des ouvrages sont alors multiples:

- contraintes géométriques pour intégrer les nouveaux ouvrages dans le tissu dense des réseaux urbains (assainissement, eau potable, chauffage urbain, câbles enterrés et canalisations diverses) et des obstacles (fondations des constructions existantes) présents dans le sous-sol urbain; - contraintes environnementales pour éviter en particulier de polluer les nappes phréatiques :

- contraintes géologiques pour limiter les déformations induites par l'excavation et pour protéger le bâti existant;

- contraintes inhérentes au système de transport (pente maximale, rayon de courbure minimal, critères de confort...).

Dans la plupart des cas, quand l'excavation doit être réalisée par des méthodes traditionnelles, un renforcement des sols est nécessaire, Le concepteur dispose d'une palette assez large de techniques de traitement de terrain, parmi lesquelles l'injection de coulis. La nette amélioration des caractéristiques mécaniques du sol générée par l'injection de produits pérennes, qui se traduit par l'appa- rition d'une cohésion et une forte augmentation de la rigidité (Biarez et al., 1998; Tailliez, 1998) n'est toutefois que trop rarement prise en compte dans les calculs de conception des ouvrages : les ouvrages de soutènement provi. soire ou définitif sont alors surdimensionnés, les délais de mise en œuvre du soutènement sont donc allongés du fait d'une plus grande pénibilité (cintres plus lourds, pourcentage d'armatures plus important), ce qui augmente le risque de décompression des terrains non injectés et le coût global des travaux. Avec le développement d'outils permettant de mieux contrôler le processus d'injection en temps réel, les injecteurs sont aujourd'hui en mesure de garantir la qualité clu traitement. Sur cette constatation, la RATP a engagé un vaste programme expérimental visant à définir des facteurs d'amélioration (rapport de la valeur d'une grandeur mécanique après injection sur sa valeur avant injection) en fonction du coulis utilisé. Dans cet article sont présentés les résultats d'essais de caractérisation des alluvions de la vallée fluviale de la Seine, d'abord vierges de tout traitement, puis injectées par un coulis de ciment ultrafin. La raison principale de ce choix est son implication immédiate pour la construction du prolongement de la ligne Météor (14* ligne du métro parisien) entre la station Madeleine et la gare Saint-Lazare (Dano, 2001).

\section{2}

\section{Description du site}

L'insertion du tunnel Météor a été délicate en rajson de la présence des lignes 3, 9, 12 et 13 du métro et des lignes A (RATP) et E (SNCF) du RER dans le soussol du quartier concerné, le $8^{e}$ arrondissement de Paris (Fig. 1) (Selosse, 1992). Le tunnel est majoritairement

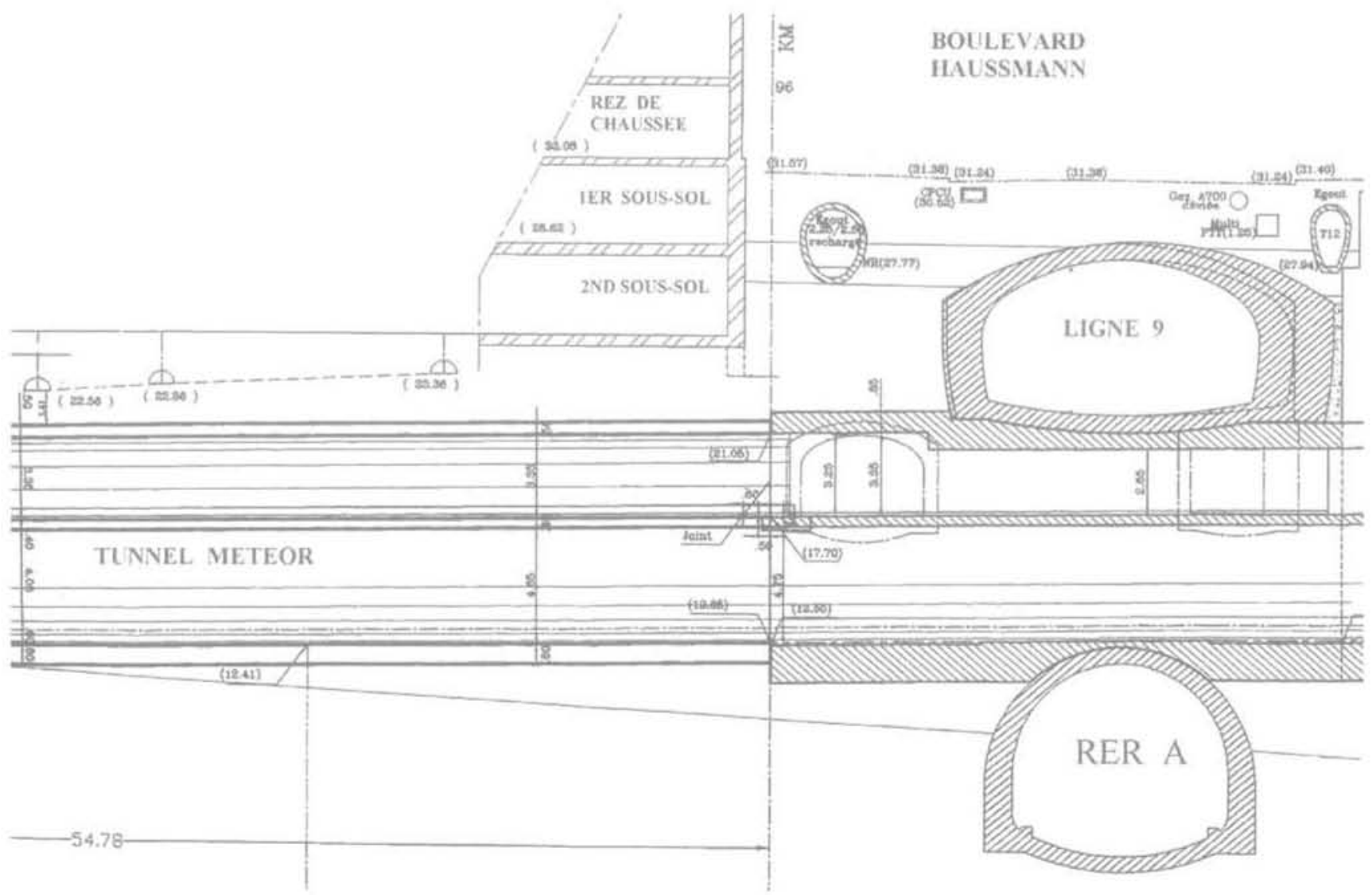

FiG 1 Implantation du tunnel Météor. Lining of the Météor Tunnel. 
implanté dans une couche de calcaire grossier et une couche de marnes et caillasses. Toutefois, sa voûte affleure dans un horizon d'alluvions anciennes dont les propriétés mécaniques se sont avéré être insuffisantes pour excaver le tunnel sans causer de désordre préjudiciable aux bâtiments sus-jacents. La voûte du tunnel effleure également les fondations d'un établissement bancaire doté de dispositifs de sécurité extrêmement sensibles. Pour ce bâtiment, les prescriptions relatives aux soulèvements induits par le traitement du terrain et aux affaissements engendrés par l'excavation sont particulièrement sévères (inférieures à 1 centimètre).

En raison de la nature du terrain et des conditions de site, l'injection de coulis a été retenue comme technique de consolidation de la couche d'alluvions anciennes. L'injection s'effectue en deux phases. Dans un premier temps, les vides les plus importants, en particulier à l'interface entre les alluvions anciennes et les marnes et caillasses, sont comblés par injection de coulis de bentonite-ciment. Les vides résiduels sont ensuite remplis par injection de coulis de ciment ultrafin. Ce traitement est associé à un rabattement de la nappe du Lutétien dont la surface libre se situe à dix mètres de profondeur environ.

Le coulis de ciment ultrafin est préféré aux gels organiques qui avaient été jusqu'alors utilisés sur les chantiers RATP mais dont l'action ne s'est pas révélée pérenne : les gels de silice se décomposent en effet par synérèse, libérant des matières polluantes pour les nappes phréatiques.

L'excavation est finalement programmée un mois après l'injection. Le comportement des sols injectés un mois après la prise du coulis est qualifié de comportement à court terme dans la suite de cet article.

\section{3}

\section{Caractérisation géotechnique des alluvions avant injection}

\section{1}

\section{Analyse granulométrique}

Des prélèvements d'alluvions anciennes ont été effectués sur l'emprise Anjou, au pied de la gare SaintLazare. Ces alluvions anciennes sont des matériaux sablo-graveleux, silico-calcaires, composés de grains sub-arrondis à sub-anguleux, dont la perméabilité en place est comprise entre $10^{-3}$ et $10^{-5} \mathrm{~m} / \mathrm{s}$ et dont l'état de compacité est moyennement dense. Après séchage, elles ont été soumises à une analyse granulométrique qui a permis de distinguer deux classes d'alluvions : les alluvions anciennes désignées par Type I et les alluvions anciennes plus grossières désignées par Type II. Mais, pour respecter un rapport minimal de 10 entre le diamètre des échantillons d'alluvions reconstitués en laboratoire et le diamètre maximal des grains du sol, leur granulométrie est écrêtée à 10 millimètres (Fig. 2), On a vérifié, a posteriori, et uniquement pour les alluvions de type I, que l'opération d'écrêtement n'a qu'une influence négligeable sur leur comportement mécanique.

Les alluvions anciennes de Type I sont alors caractérisées par un coefficient d'uniformité $C_{u}$ égal à 2,1 et

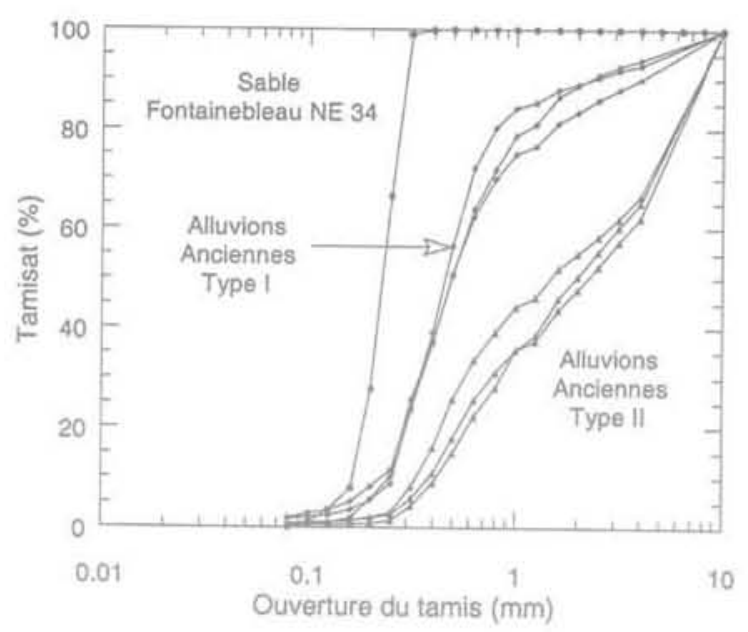

FIG.2 Granulométrie des sols après écrêtement. Grading curves of soils after reduction of the maximal grain diameter.

un diamètre moyen des grains $\mathrm{D}_{50}$ égal à $410 \mu \mathrm{m}$, tandis que, pour les alluvions anciennes de Type II, le coefficient d'uniformité et le diamètre moyen des grains valent respectivement 5,9 et $1300 \mu \mathrm{m}$. Leurs poids volumiques minimal $\gamma_{\min }$ et maximal $\gamma_{\text {mix }}$, déterminés grâce à un dispositif conforme à la normalisation française (NF P 94-059) (Kim, 1995), valent respectivement $14,9 \mathrm{kN} / \mathrm{m}^{3}$ et $17,9 \mathrm{kN} / \mathrm{m}^{9}$ pour les alluvions de Type I, $16,4 \mathrm{kN} / \mathrm{m}^{3}$ et $19,4 \mathrm{kN} / \mathrm{m}^{3}$ pour les alluvions de Type II (Tableau 1). Les données $\gamma_{\min }$ et $\gamma_{\max }$ permettent de calculer l'indice de densité relative $\mathrm{I}_{d}$ d'un sol :

$$
I_{d}(\%)=\frac{\gamma_{\max }}{\gamma} \times \frac{\gamma-\gamma_{\min }}{\gamma_{\max }-\gamma_{\min }} \times 100
$$

TAELEAUI Caractéristiques des sols étudiés après écrêtement. Characteristics of soils.

\begin{tabular}{|c|c|c|c|c|}
\hline $\begin{array}{l}\text { SOL } \\
\text { Unités. }\end{array}$ & $\mathrm{C}_{\text {H }}$ & $\begin{array}{l}\mathrm{D}_{\text {set }} \\
(\mu \mathrm{m})\end{array}$ & ${ }_{(k N / m y}$ & $\left(\mathrm{kN} / \mathrm{m}^{2}\right)$ \\
\hline Sable de Fontainebleau NE34 & 1,4 & 220 & 14.0 & 16,5 \\
\hline Alluvions anciennes de Type I & 2,1 & 410 & 14,9 & 17,9 \\
\hline Alluvions anciennes de Type II & 5,9 & 1300 & 16,4 & 19,4 \\
\hline
\end{tabular}

Un sable de référence, en l'occurrence le sable de Fontainebleau NE34, fin et siliceux, a également été retenu. Disponible en grande quantité, contrairement aux alluvions, le sable de Fontainebleau a été utilisé pour réaliser des campagnes d'essais plus approfondies sur les sols injectés pour mettre en évidence les facteurs prépondérants sur leur comportement mécanique et pour établir des corrélations empiriques liant la résistance en compression simple à ces facteurs clés.

\section{2}

\section{Essais de caractérisation}

Les sols pulvérulents ont ensuite été soumis à des essais triaxiaux consolidés drainés (Normes françaises P94-070, P 94-074) dans une cellule triaxiale. Les échantillons, de $100 \mathrm{~mm}$ de diamètre et de $200 \mathrm{~mm}$ de hau- 


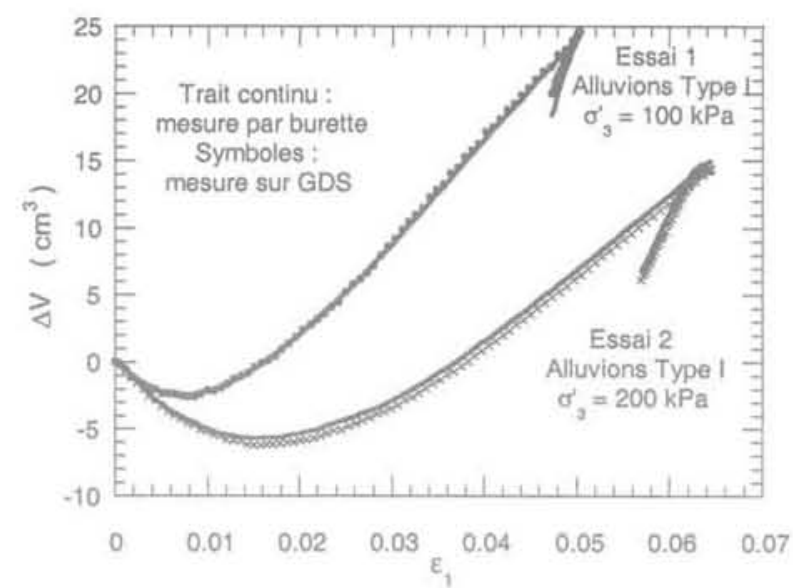

FIQ.3 Équivalence de la mesure des variations de volume par lecture sur une burette graduée ou sur le contrôleur pression-volume. Equivalence of the measure of volumetric variations by reading on a gracluated burette or on the volume-pressure controller.

teur, sont reconstitués soit par pluviation dans le cas du sable de Fontainebleau, soit manuellement dans le cas des alluvions, la dimension des grains ne permettant une mise en place par pluviation. Le matériau alluvionnaire est ainsi déposé en cinq couches. Chacune de ces couches est compactée par 30 coups de dame. La repro-
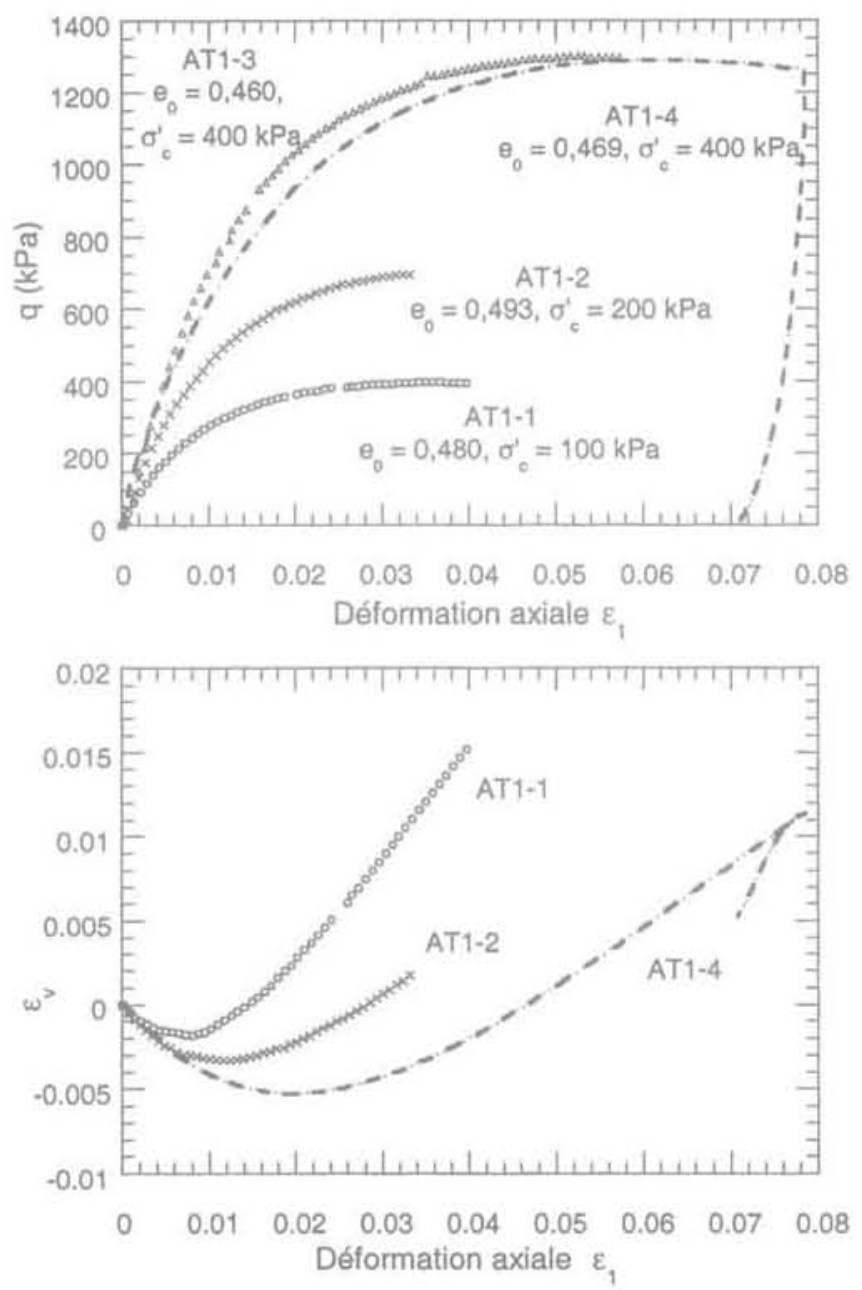

मG. 4 Essais triaxiaux sur les alluvions de Type I. Triaxial tests on Type I alluvial deposits. ductibilité de ces deux méthodes (pluviation, remplissage manuel) est satisfaisante. Les indices des vides en fin de consolidation sont compris entre 0,46 et 0,49 pour les alluvions de Type I (soit un indice de densité relative $I_{4}$ proche de $85 \%$ ), entre 0,37 et 0,40 pour les alluvions de Type II (soit un indice de densité relative $I_{d}$ proche de $80 \%$ ) et entre 0,60 et 0,62 pour le sable de Fontainebleau (soit un indice de densité relative $\mathrm{I}_{d}$ de $85 \%$ ).

Les contraintes latérales $\sigma_{3}$, appliquées grâce à un contrôleur pression-volume GDS Standard, varient entre 100 et $400 \mathrm{kPa}$. Les déformations volumiques sont mesurées soit à partir des variations de volume dans une burette graduée reliée à l'échantillon lorsque les échantillons sont saturés, soit à partir des variations de volume enregistrées sur le contrôleur pression-volume, pourvu qu'elles soient corrigées de l'enfoncement du piston de chargement. On montre expérimentalement, sur des échantillons de sable saturés, que ces deux mesures sont équivalentes (Fig. 3). Les variations de hauteur de l'échantillon sont lues sur un comparateur extérieur à la cellule. Le capteur de force, d'une capacité de $5 \mathrm{kN}$, est immergé dans la cellule. Les échantillons sont cisaillés à une vitesse de déformation de $1,28 \times 10^{-5} \mathrm{~s}^{-1}$, pour des contraintes latérales successives de 100,200 et $400 \mathrm{kPa}$.

Les résultats des essais sont présentés sur la figure 4 pour les alluvions de Type I, sur la figure 5 pour les alluvions de Type II et sur la figure 6 pour le sable de Fontainebleau.
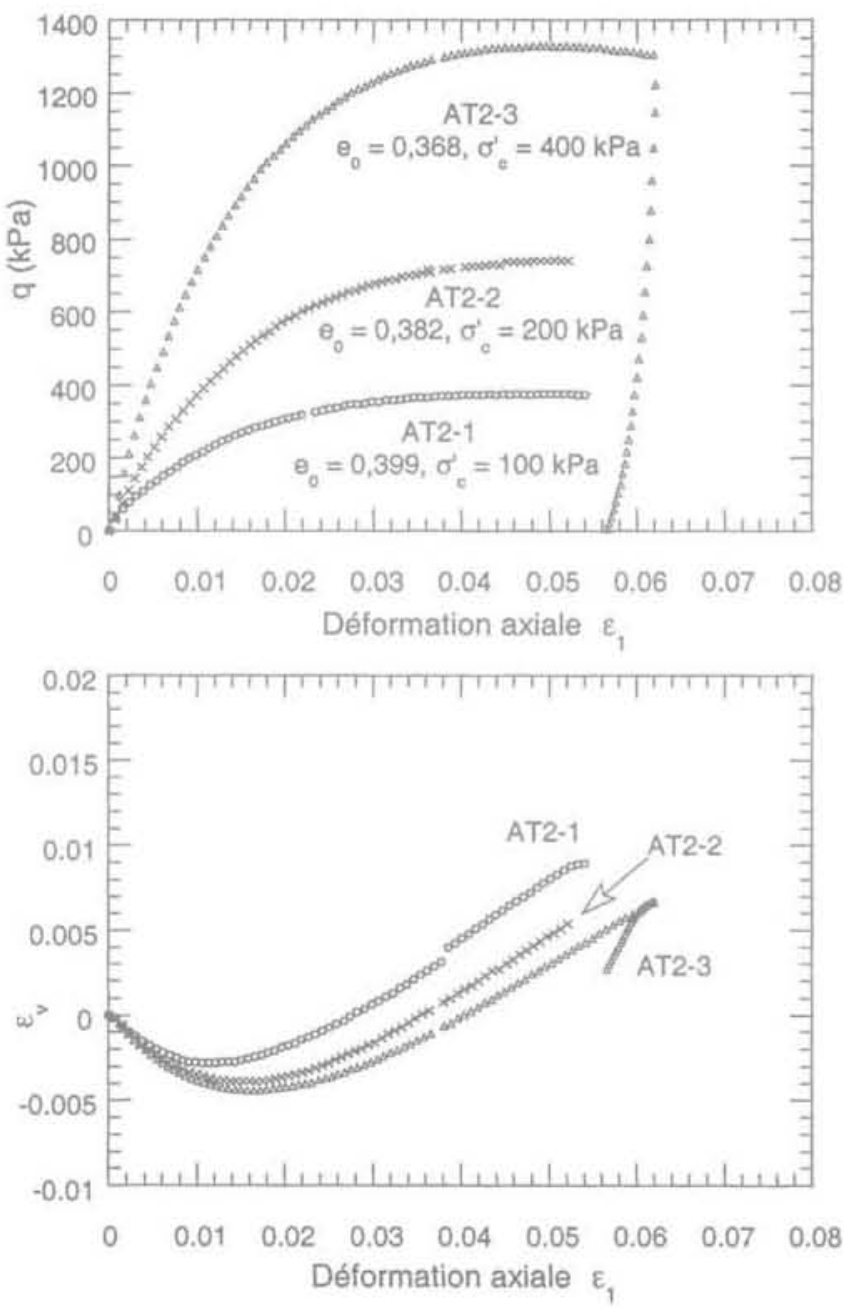

FIG.5 Essais triaxiaux sur les alluvions de Type II. Triaxial tests on Type II alluvial deposits. 

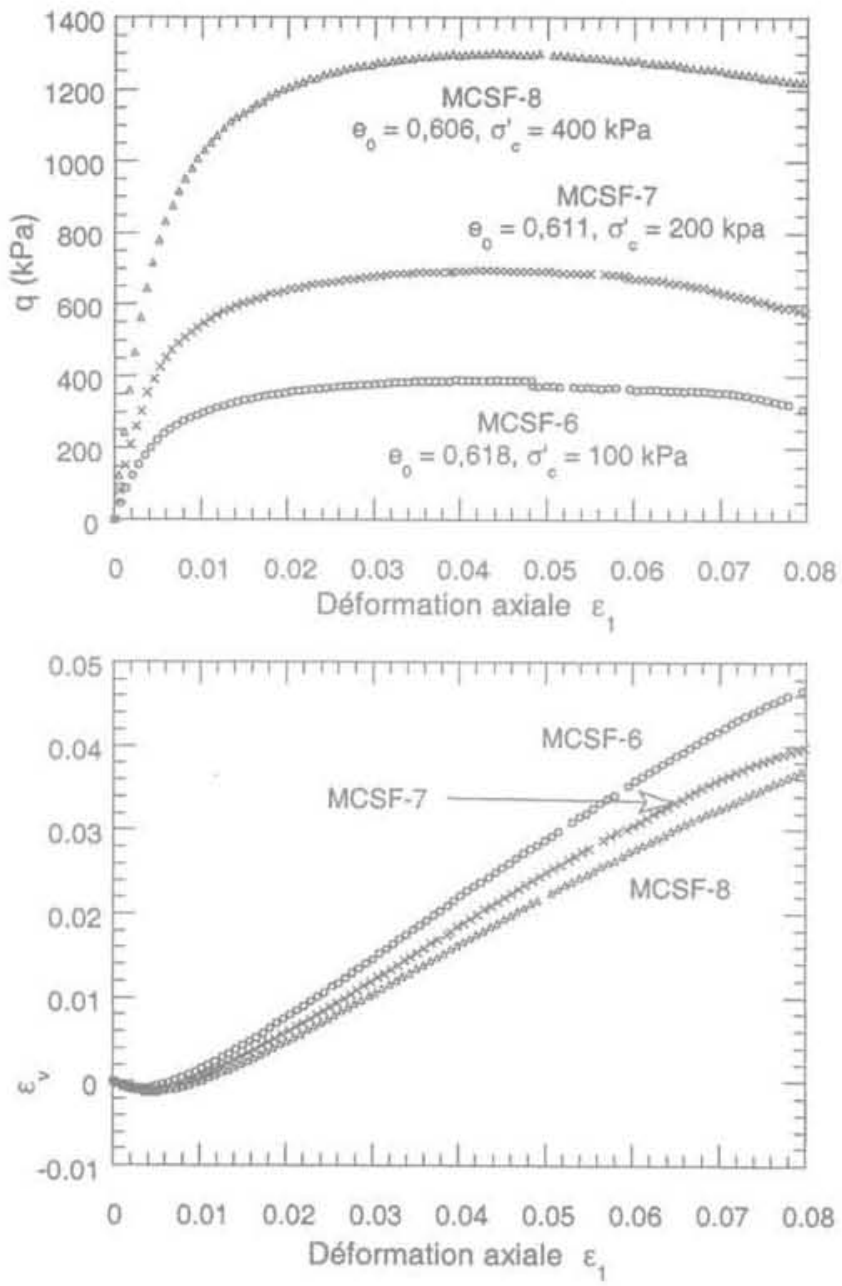

FG. 6 Essais triaxiaux sur le sable de Fontainebleau NE34.

Triaxial tests on Fontainebleau Sand NE34.

$$
\sin \Psi=\frac{\Delta \varepsilon_{\mathrm{v}} / \Delta \varepsilon_{1}}{2+\Delta \varepsilon_{\mathrm{v}} / \Delta \varepsilon_{1}} \quad \Delta \varepsilon_{1} \geq 0, \Delta \varepsilon_{\mathrm{v}} \geq 0
$$

- la cohésion $\mathrm{c}^{\prime}$;

- l'angle de frottement interne $\varphi^{\prime}$.

Ces deux derniers paramètres sont déterminés soit dans le plan de Mohr-Coulomb, soit dans le plan ( $\left.\mathrm{p}^{\prime}, \mathrm{q}\right)$ à partir des relations (4a) et (4b) dans lesquelles interviennent les paramètres $M_{n}$ et $C_{m}$ dont la signification est donnée sur la figure 7 :

$$
\begin{aligned}
& \varphi_{p}^{\prime}=\operatorname{Arcsin}\left(\frac{3 M_{p}}{6+M_{p}}\right) \\
& c^{\prime}=\frac{\left(3-\sin \varphi_{p}^{\prime}\right)}{6 \cos \varphi_{p}^{\prime}} C_{m}
\end{aligned}
$$

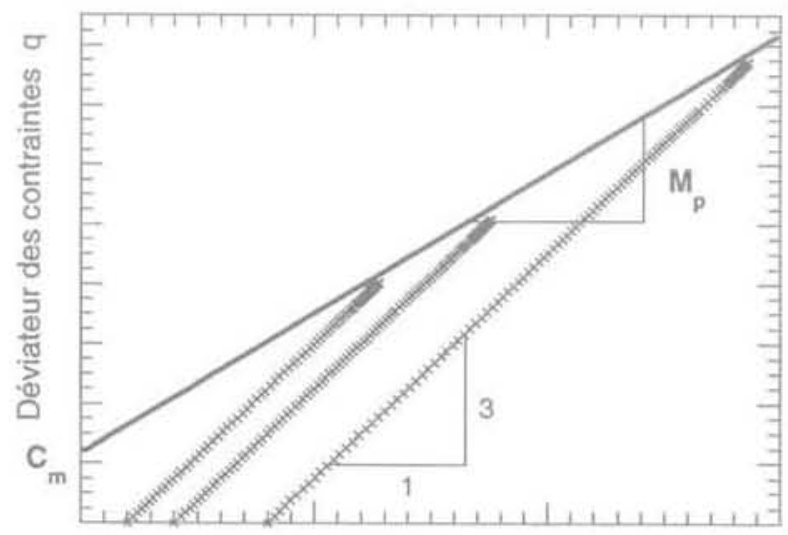

Contrainte moyenne effective $p^{\prime}$

FG.7 Identification des paramètres de résistance maximale.

Identification of the maximal shear strength parameters.

\section{Interprétation des essais}

Le comportement des trois sols étudiés est caractéristique du comportement des sols pulvérulents. On choisit donc d'interpréter ces essais de manière conventionnelle, en identifiant les cinq paramètres du modèle élastique plastique parfait, en faisant l'hypothèse d'un critère de Mohr-Coulomb non associé. Ces cinq paramètres sont successivement :

- le module sécant à l'origine de la courbe déviateur des contraintes/déformation axiale pour une déformation de $10^{-3}$.

$$
\mathrm{E}_{\mathrm{sec}}\left(\varepsilon=10^{-3}\right)=\frac{\Delta \mathrm{q}}{\Delta \varepsilon_{1}}
$$

- le coefficient de Poisson v déterminé à l'origine de la courbe déformation volumique/déformation axiale. dans sa partie contractante :

$$
v=\frac{1}{2}\left(1+\frac{\Delta \varepsilon_{v}}{\Delta \varepsilon_{1}}\right) \quad \Delta \varepsilon_{1} \geq 0, \Delta \varepsilon_{v} \leq 0
$$

- l'angle de dilatance $\psi$ calculé dans le domaine dilatant au point d'inflexion de la courbe des déformations volumiques :

\section{4}

\section{Valeurs des paramètres du modèle élastique plastique parfait}

Les différences entre les deux types d'alluvions anciennes sont relativement peu marquées (Tableau II). Ainsi, les valeurs du module sécant varient, en fonction de la contrainte latérale $\sigma_{3}^{\prime}$, entre $46 \mathrm{MPa}$ et $126 \mathrm{MPa}$ pour les alluvions de Type I, et entre $47 \mathrm{MPa}$ et $110 \mathrm{MPa}$ pour les alluvions de Type II. Les valeurs obtenues pour le sable de Fontainebleau sont plus élevées et comprises entre 64 et $222 \mathrm{MPa}$. L'augmentation du module sécant en fonction de la contrainte $\sigma_{3}^{\prime}$ est presque linéaire, ceci en raison du niveau de déformation considéré pour la mesure de ce paramètre $\left(\varepsilon=10^{-3}\right)$. Par ailleurs, les valeurs du coefficient de Poisson sont classiquement proches de 0,2 ,

L'angle de dilatance semble être le paramètre le plus affecté par la nature et la granulométrie du sol étudié : plus le coefficient d'uniformité C des sols est important, plus les valeurs de l'angle de dilatance sont faibles. Cette constatation expérimentale traduit la 
TABLEAU II Paramètres des sols avant injection.

Parameters of the elastic perfectly plastic model before injection.

\begin{tabular}{|c|c|c|c|c|c|c|c|}
\hline $\mathrm{E}_{\mathrm{m} \text { ( }}(\mathrm{MPa})$ & $\sigma_{3}=100 \mathrm{kPa}$ & $\sigma_{3}^{\prime}=200 \mathrm{kPa}$ & $\sigma^{\prime}=400 \mathrm{kPa}$ & v & $\sigma^{\prime}=100 \mathrm{kPa}$ & $\sigma_{2}^{\prime}=200 \mathrm{kPa}$ & $\sigma_{3}=400 \mathrm{kPa}$ \\
\hline SF NE34 & 64 & 131 & 222 & SF NE34 & 0,24 & 0,25 & 0,20 \\
\hline AA Type I & 46 & 71 & 126 & AA Type I & 0,16 & 0,12 & 0,22 \\
\hline AA Type II & 47 & 68 & 110 & AA Type II & 0,23 & 0,20 & 0,22 \\
\hline$\psi(9)$ & $\sigma_{3}=100 \mathrm{kPa}$ & $\sigma_{s}^{\prime}=200 \mathrm{kPa}$ & $\sigma_{1}=400 \mathrm{kPa}$ & & $M_{p}$ & $\varphi(9)$ & $c^{\prime}(\mathrm{kPa})$ \\
\hline SF NE 34 & 15,6 & 14,1 & 13,1 & SF NE34 & 1,60 & 39,1 & 0 \\
\hline AA Type I & 14,6 & 9.8 & 8,9 & AA Type I & 1,49 & 36,6 & 0 \\
\hline AA Type II & 8,9 & 8,3 & 7,8 & AA Type II & 1,54 & 37.8 & 0 \\
\hline
\end{tabular}

capacité de désenchevêtrement moins importante pour un squelette granulaire contenant des particules de plus grand diamètre.

Les valeurs des angles de frottement interne pour les alluvions de Type I et de Type II sont respectivement de 36,6 degrés $\left(M_{p}=1,49\right)$ et de 37,8 degrés $\left(M_{p}=\right.$ 1,54). Pour le sable de Fontainebleau, I'angle de frottement interne est de 39,1 degrés (Tableau II). La cohésion est nulle dans les trois cas.

\section{4}

\section{Caractérisation géotechnique des alluvions après injection}

On aurait pu étudier le comportement mécanique des sols injectés prélevés in situ. Cependant, la présence de granulats de taille relativement importante par rapport aux dimensions des éprouvettes pose un problème évident de représentativité de ces échantillons. Les éprouvettes de sol injecté ont donc été préparées en laboratoire, à partir des matériaux dont la granulométrie a été écrêtée. Ceci permet d'avoir un contrôle sur les différentes étapes du processus d'injection, depuis la mise en place du sol jusqu'à la réalisation des essais triaxiaux.

\section{1}

\section{Reconstitution en laboratoire des éprouvettes de sol injecté}

Le dispositif d'injection se compose d'un tube en plexiglas, rigide et transparent, de $100 \mathrm{~mm}$ de diamètre et de $900 \mathrm{~mm}$ de hauteur (Fig. 8) (Tailliez, 1998; Dano, 2001). Le sol est introduit par couches successives de dix centimètres d'épaisseur environ, compactées par trente coups de marteaux portés sur l'enveloppe en Plexiglas. L'indice de densité relative finalement obtenu est compris entre 90 et $100 \%$. En modifiant le nombre et l'intensité des coups de marteaux portés sur la colonne, on a pu obtenir des colonnes de sol de densité plus faible. Si l'homogénéité est globalement respectée, la reproductibilité, par contre, dépend alors de I'habileté de l'expérimentateur.

Le coulis, mélange de ciment ultrafin (le diamètre maximal des grains de ciment est inférieur à $12 \mu \mathrm{m}$ ). d'eau, d'une charge inerte et d'un adjuvant qui assurent la stabilité et la fluidité du coulis pendant l'injection, est préparé selon une procédure qui lui est propre. On mesure, à des fins de contrôle, sa densité et sa viscosité. Celles-ci dépendent du rapport massique ciment/eau de gâchage noté $\mathrm{C} / \mathrm{E}$.

Maintenu constamment en agitation pour conserver son homogénéité, le coulis est injecté à débit constant $\left(6 \mathrm{~cm}^{3} / \mathrm{s}\right)$ dans la colonne. Pendant l'injection, on enregistre l'évolution de la masse de coulis, de la pression d'injection et de la position du front d'injection: ces trois quantités, lorsqu'elles évoluent de manière linéaire dans le temps, attestent de l'homogénéité de la colonne de sol (Dano, 2001). L'injection est arrêtée lorsque suffisamment de coulis est recueilli à la sortie de la colonne.

Les colonnes sont alors stockées verticalement, pendant au moins 28 jours (durée correspondant aux planning d'exécution des travaux in situ), en chambre humide à température et hygrométrie contrôlées. Les profils de densité obtenus par gammadensimétrie (Fig.9) confirment l'homogénéité des colonnes: la

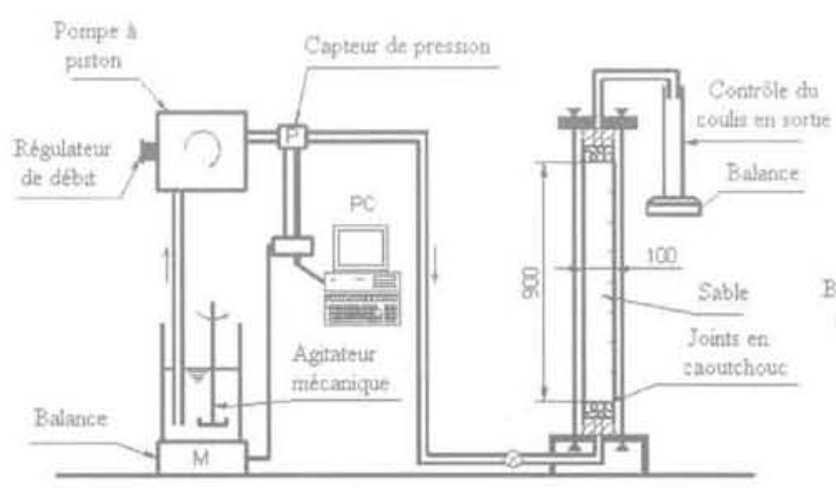

DISPOSTIF D' INJECTION

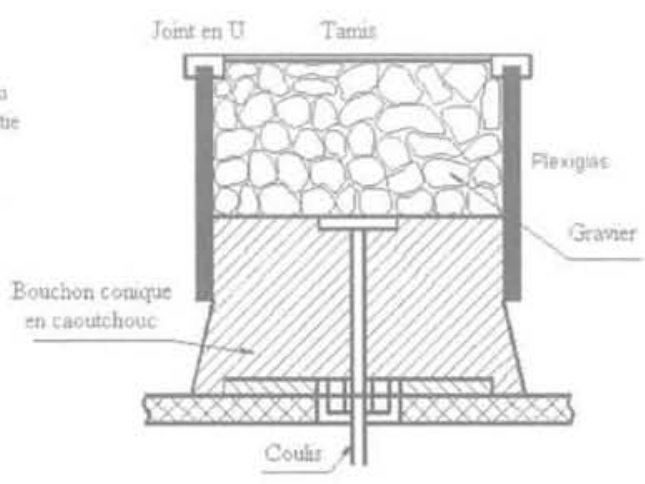

DETAII 


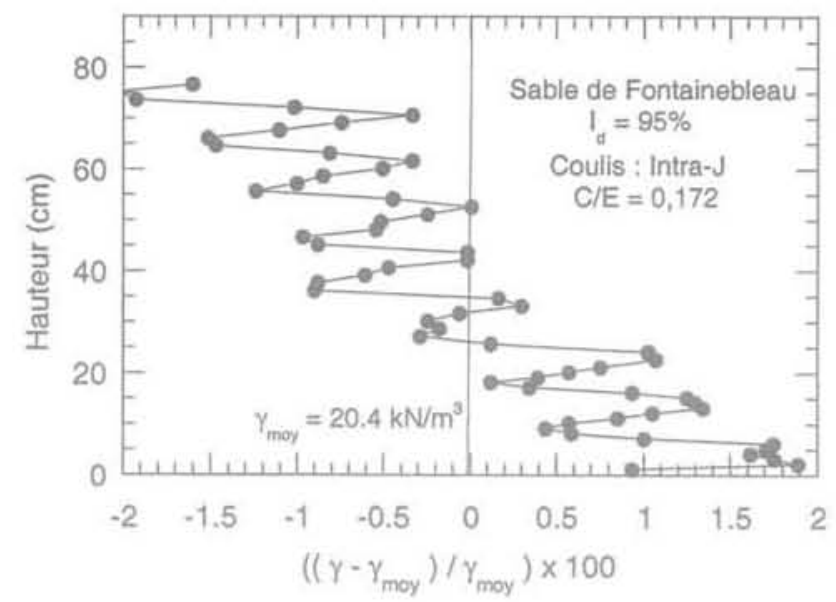

F16.9 Profil de densité sur une colonne de sable de Fontainebleau obtenu par gammadensimétrie.

Density profile on a sample of grouted Fontainebleau sand by gammadensimetry.

variation du poids volumique rapporté au poids volumique moyen n'excède pas $4 \%$ sur toute la hauteur de la colonne. Les irrégularités dans le profil sont liées au mode de dépôt du sol par couches de $10 \mathrm{~cm}$.

Finalement, on découpe, par sciage, dans chaque colonne, trois éprouvettes dont les faces sont ensuite rectifiées.

\section{2}

\section{Essais de caractérisation}

Ces éprouvettes sont ensuite soumises à des essais de caractérisation menés sous la forme de deux campagnes d'essais distinctes :

- une première campagne d'essais de résistance en compression simple. Ce type d'essai est couramment utilisé pour évaluer l'efficacité du traitement d'injection. Le but principal est de proposer une corrélation empirique d'évolution de la résistance en compression simple $\mathrm{R}_{c}$ en fonction du sol et du coulis :

- une seconde campagne d'essais triaxiaux pour contrôler l'effet des facteurs clés sur une gamme plus importante de contraintes et pour identifier les valeurs des paramètres du modèle élastique plastique parfait.

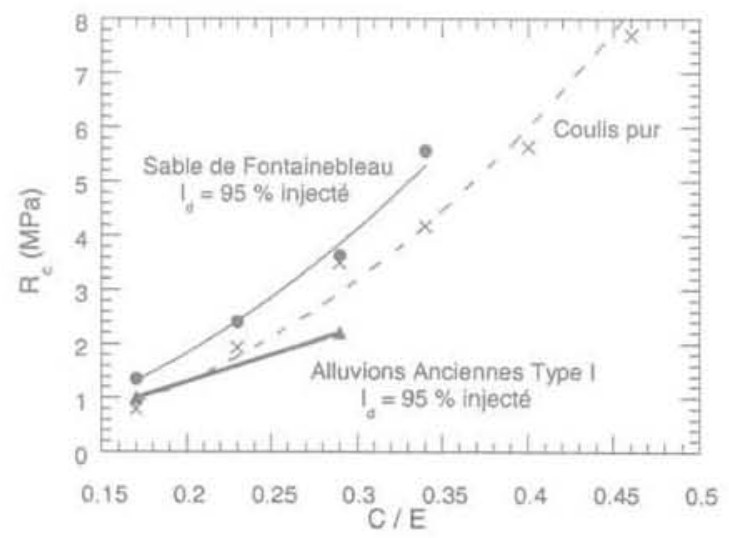

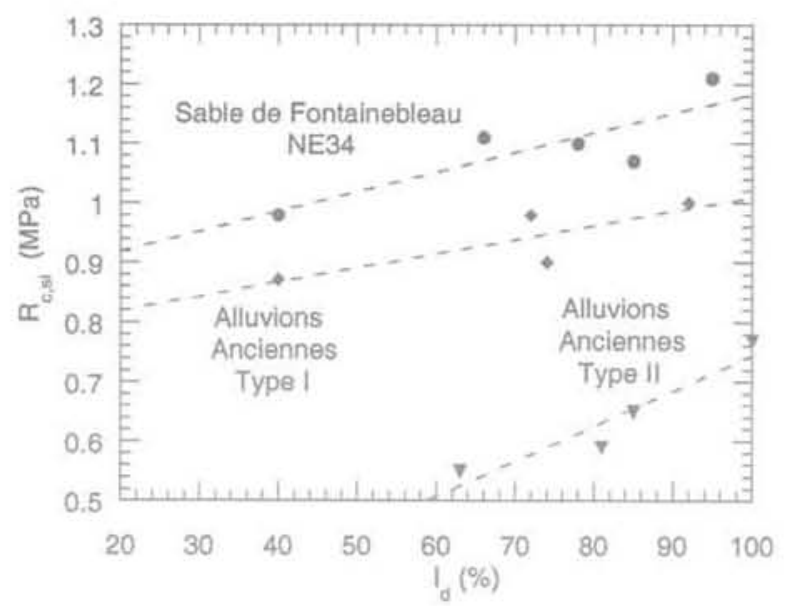

fiG.10 Évolution de la résistance en compression simple en fonction de l'indice de densité relative pour les 3 sols étudiés. Evolution of the unconfined compressive strength as a function of the relative density index for the 3 tested soils.

\section{3}

\section{Première campagne d'essais : essais de compression simple}

Sur la base de publications relatives au comportement des mélanges sable/liant, on constate que la résistance en compression simple de ces mélanges dépend de facteurs clés tels que :

- la nature du liant et sa teneur en ciment (Dupas et Pecker, 1979; Tailliez, 1998) ;

- la granulométrie, la densité du sol et la morphologie des particules (Clough et al., 1981 ; Zebovitz et al., 1989; Airey, 1993 ; Bennabi et Levacher, 1995 ; Baig et al., 1997; Huang et Airey, 1998) ;

- la minéralogie des grains du sol (Ismail et al., 2000); - l'état de saturation initial du sol : les sols injectés à sec présentent une résistance plus élevée que les sols injectés préalablement saturés en eau (Bennabi et Levacher, 1995 ; Perret et al., 2000) ;

- le coefficient de remplissage des pores par le coulis (Schwarz et Krizek, 1994a, b).

Parmi tous ces facteurs, certains ont un effet plus important que d'autres sur la résistance en compression simple. Il en est ainsi de la teneur en liant et de la

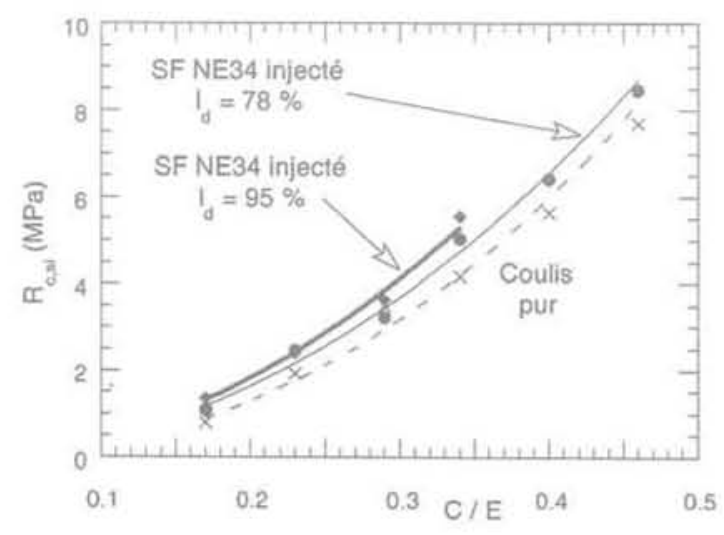

FlG. 11 Évolution de la résistance en compression simple en fonction du rapport $\mathrm{C} / \mathrm{E}$ et de l'indice $\mathrm{I}_{\mathrm{d}}$. Evolution of the unconfined compressive strength as a function of the ratio $\mathrm{C} / \mathrm{E}$ and the index $\mathrm{I}_{\mathrm{a}}$. 
densité du squelette granulaire. Ceci s'explique par le fait qu'une compacité forte favorise la multiplication des contacts intergranulaires et une diminution de la porosité. Or les grains de ciment du coulis se déposent préférentiellement aux points de contact entre les grains et sont piégés d'autant plus facilement que la dimension des pores est faible. La cimentation s'initie en ces points de contact et aboutit à un enrobage des grains du sol d'autant meilleur que la quantité de liant introduite est également plus importante (Arenzana et al., 1989). La qualité de l'encastrement des particules dans la matrice de liant conditionne la résistance finale (Bennabi et Levacher, 1995).

Un certain nombre d'essais de résistance en compression simple a alors permis de préciser l'effet du rapport $\mathrm{C} / \mathrm{E}$ du coulis, de l'état de compacité du sol (par le biais de l'indice de densité relative Id), de la nature et de la granulométrie du sol. Le coefficient de remplissage des pores, estimé à partir du suivi de l'injection (Dano, 2001), est, dans tous les cas, proche de $100 \%$.

On représente, sur la figure 10, l'évolution de la résistance en compression simple en fonction de l'indice de densité relative $I_{d}$ pour les trois sols injectés par le même coulis de rapport $\mathrm{C} / \mathrm{E}=0,172$. Pour cette valeur, la résistance en compression simple du coulis pur vaut $0,8 \mathrm{MPa}$. La résistance en compression simple des éprouvettes de sol injecté croît, en première approximation, de manière linéaire en fonction de l'indice de densité relative du sol. Toutefois, les pentes d'évolution dépendent de la nature et de la granulométrie du sol. Kaga et Yonekura (1991) trouvent des résultats similaires pour différents sables injectés au gel de silice et précisent que la pente de la droite $R_{c}=f\left(I_{d}\right)$ est fonction du coulis et de la surface spécifique du sol.

Les résistances les plus grandes, comme le laisse entrevoir la littérature (Saxena et Lastrico, 1978; Zebovitz et al., 1989), sont obtenues pour le sable de Fontainebleau dont le diamètre moyen des particules est le plus faible. La résistance en compression simple croît également lorsqu'on augmente le rapport C/E (Fig. 11). Mais son évolution n'est plus linéaire dans l'intervalle des valeurs étudiées, c'est-à-dire entre 0,17 et 0,44 (Fig. 11). Sur cette figure, on reporte les résultats d'essais de compression simple réalisés sur des éprouvettes de coulis pur, sur des éprouvettes de sable de Fontainebleau d'indice de densité relative égal à $78 \%$ et sur des éprouvettes de sable de Fontainebleau d'indice de densité relative égal à $95 \%$. Les données expérimentales concernant le coulis ont été approchées par une loi en puissance telle que :

$$
R_{\mathrm{Cc}}(\mathrm{MPa})=\lambda_{\mathrm{c}}\left(\frac{\mathrm{C}}{\mathrm{E}}\right)^{N_{\mathrm{c}}}
$$

où $\mathrm{R}$ est la résistance en compression du coulis pur exprimée en $\mathrm{MPa}, \lambda$ et $\mathrm{N}$ deux constantes d'ajustement. Pour le coulis étudié :

$$
\lambda_{c}=45,3 \mathrm{MPa} \text { et } \mathrm{N}_{c}=2,21
$$

On admet que la résistance en compression $\mathrm{R}_{\text {cis }}$ des éprouvettes de sol injecté suit également une loi en puissance telle que :

$$
R_{c, s l}=\lambda_{s}\left(I_{d}, \text { sol, coulis }\right) \times\left(\frac{C}{E}\right)^{N_{s}\left(I_{d} \cdot \text { sol, coutis }\right)}
$$

où $\lambda_{\mathrm{s}}$ dépend de la densité et des caractéristiques morphologiques des grains du sol. On propose la relation :

$$
\lambda_{\mathrm{s}}=\lambda_{\mathrm{c}}+\lambda_{\mathrm{g}}\left(\mathrm{I}_{\mathrm{d},} \mathrm{sol}\right)
$$

On suppose aussi que l'exposant $\mathrm{N}$, qui caractérise la loi d'évolution de la résistance du sol injecté est relié à l'indice de densité relative $I_{d}$ par la relation (Kaga et Yonekura, 1991) :

$$
\mathrm{N}_{\mathrm{s}}=\mathrm{N}_{\mathrm{s0}}-\mathrm{N}_{\mathrm{s1}} \times \mathrm{I}_{\mathrm{d}}
$$
que

Par identification avec le coulis pur $\left(\mathrm{I}_{d}=0\right)$, on établit

$$
\mathrm{N}_{\mathrm{s0}}=\mathrm{N}_{\mathrm{c}}
$$

D'où, finalement, la corrélation suivante :

$$
R_{c, s i}=\left(\lambda_{c}+\lambda_{g}\left(\text { sol }, I_{d}\right)\right) \times\left[\frac{C}{E}\right]^{N_{c}-N_{s t} l_{d}}
$$

Les essais de compression simple réalisés sur le sable de Fontainebleau d'indice de densité relative $78 \%$ puis $95 \%$ concluisent ainsi aux corrélations suivantes :

$$
\begin{aligned}
& -I_{d}=78 \%: \quad R_{c, s i}\left(I_{d}=78 \%\right)=45,8 \times\left[\frac{C}{E}\right]^{2.00} \\
& -I_{d}=95 \%: \quad R_{c s i}\left(I_{d}=95 \%\right)=41,2 \times\left[\frac{C}{E}\right]^{2.00}
\end{aligned}
$$

Toutefois, les essais de résistance en compression simple ne sont en général pas suffisants pour avoir une idée précise sur l'influence des facteurs clés. Les essaís triaxiaux présentés ci-dessous permettent de répondre à l'influence de la contrainte moyenne sur le comportement des sols injectés.

\section{4}

\section{Seconde campagne d'essais : essais triaxiaux}

Ces essais triaxiaux, consolidés et drainés, sans saturation préalable des échantillons, ont été effectués avec une vitesse de déformation de $8,4 \times 10^{-6} \mathrm{~s}^{-1}$ sur des éprouvettes :

- de sable de Fontainebleau NE $34\left(I_{4}=95 \%\right)$ injecté par le coulis de rapport $\mathrm{C} / \mathrm{E}=0,172$ puis 0,235 (Fig. 12a, b) :

- d'alluvions anciennes de Type I $\left(I_{d}>95 \%\right)$ injectées par le coulis de rapport $\mathrm{C} / \mathrm{E}=0,172$ (Fig. 13) ;

- d'alluvions anciennes de Type II $\left(\mathrm{I}_{\mathrm{d}}>95 \%\right)$ injectées par le coulis de rapport $\mathrm{C} / \mathrm{E}=0,172$ (Fig. 14).

On compare sur la figure 15 le comportement des alluvions anciennes de Type I avant et après injection. Ces essais illustrent d'une manière générale l'effet de l'injection sur le comportement des sols. On note ainsi la nette amélioration apportée par le coulis tant sur la rigidité initiale que sur la résistance maximale.

L'effet de la contrainte moyenne sur la rigidité initiale semble par ailleurs plus faible dans le cas des sols injectés que dans le cas des sols vierges de tout traitement. Par contre, l'évolution du déviateur maximal avec la contrainte appliquée signifie que le caractère frottant du comportement, lié au squelette granulaire, n'est pas effacé par l'injection.

Ces figures montrent également que le comportement des sols injectés est de type contractant-dilatant. Une différence fondamentale apparait cependant: le taux de dilatance est nettement plus élevé dans le cas des sols injectés. L'angle de dilatance mesuré lors d'un essai sur une éprouvette de sol injecté, comme on le verra plus loin, est toujours supérieur ou égal à l'angle de dilatance identifié à partir d'un essai sur le sol vierge 

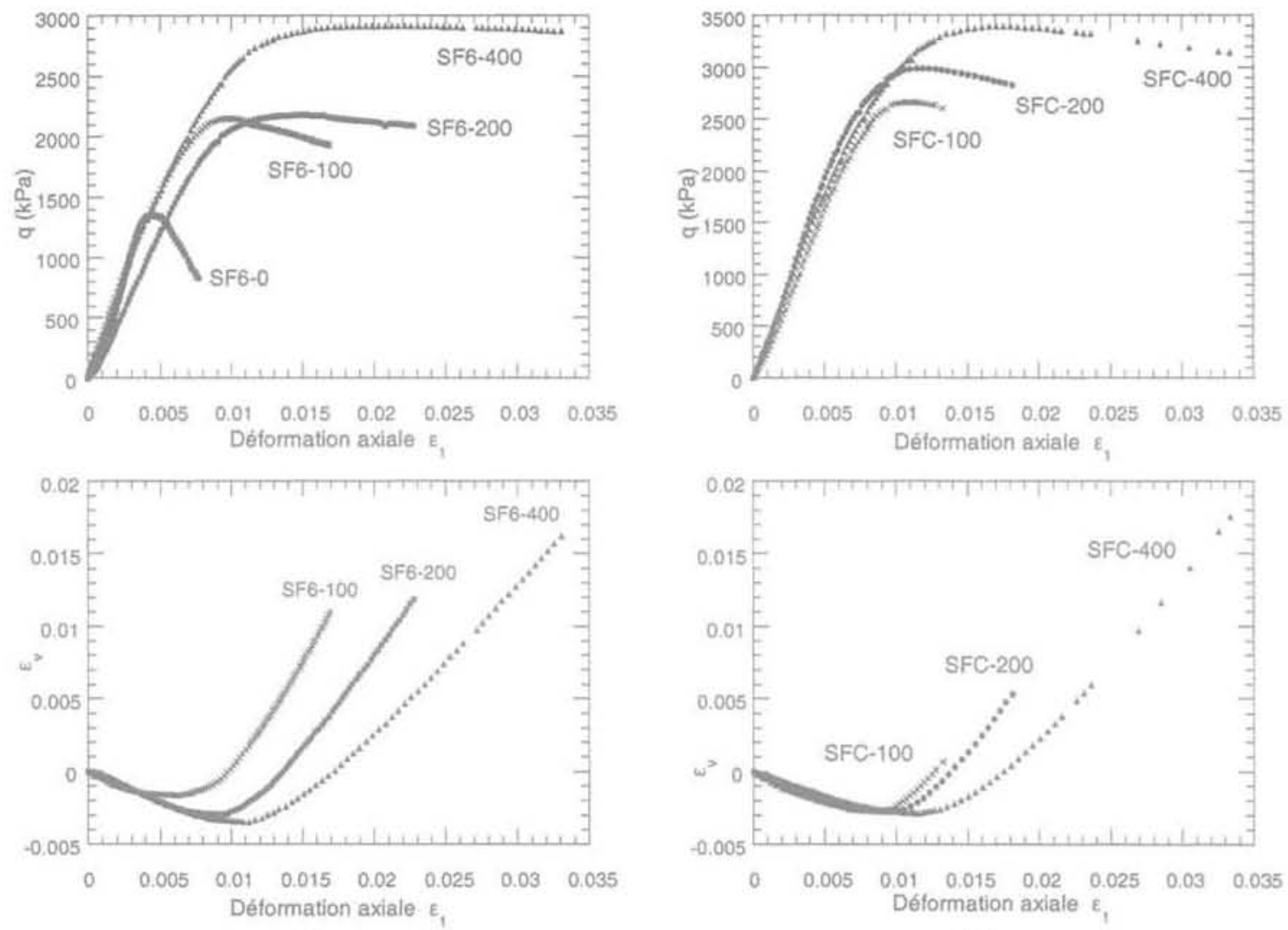

(a)

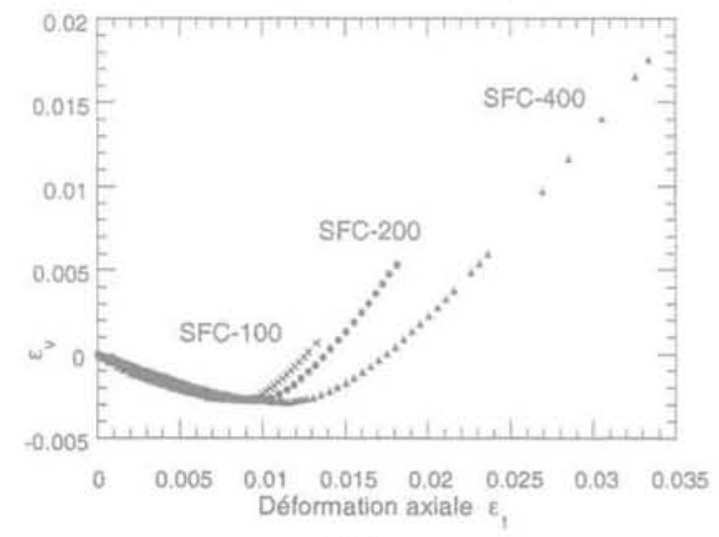

(b)

FG.12 (a) Essais triaxiaux sur le sable de Fontainebleau injecté par le coulis de rapport $\mathrm{C} / \mathrm{E}=0,172$.

(b) Essais triaxiaux sur le sable de Fontainebleau injecté par le coulis de rapport $\mathrm{C} / \mathrm{E}=0,235$.

(a) Triaxial tests on the Fontainebleau Sand injected by the grout with a ratio $\mathrm{C} / \mathrm{E}=0.172$.

(b) Triaxial tests on the Fontainebleau Sand injected by the grout with a ratio $\mathrm{C} / \mathrm{E}=0.235$.
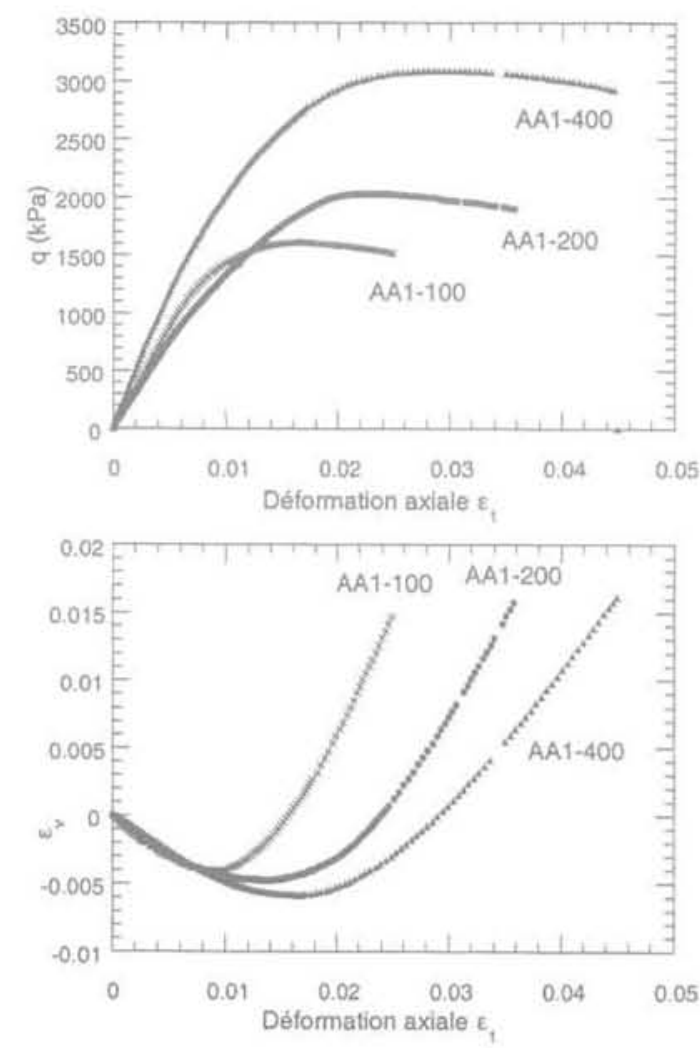

FG. 13 Essais triaxiaux sur les alluvions anciennes de Type 1 injectées. Triaxial tests on grouted Type I alluvial deposits.
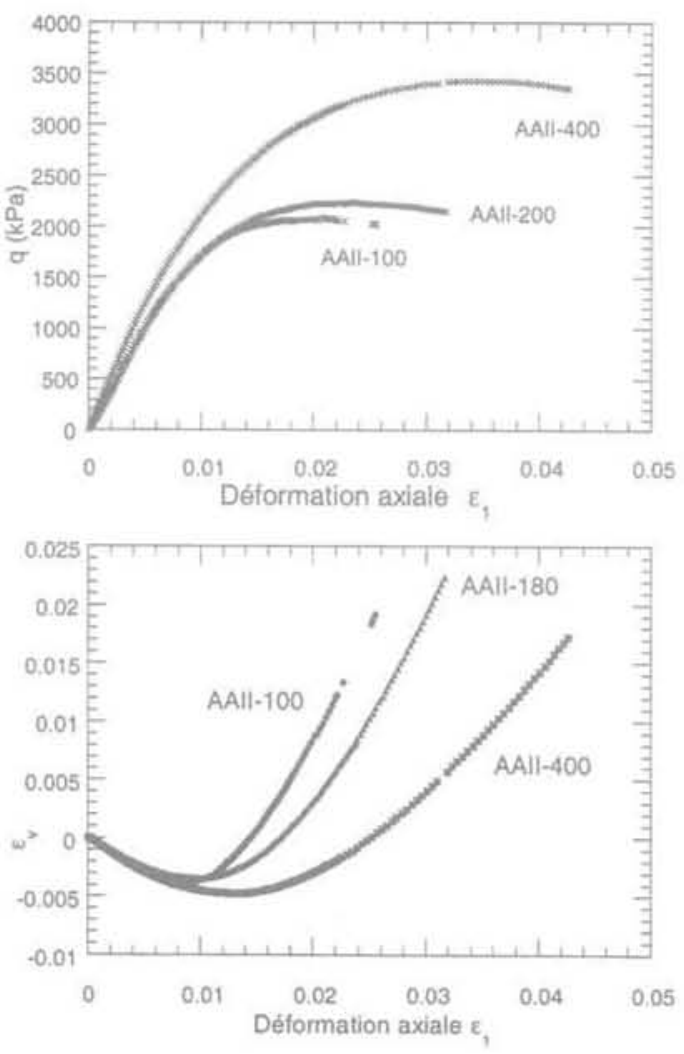

FG. 14 Essais triaxiaux sur les alluvions anciennes de Type Il injectées. Triaxial tests on grouted Type II alluvial deposits. 

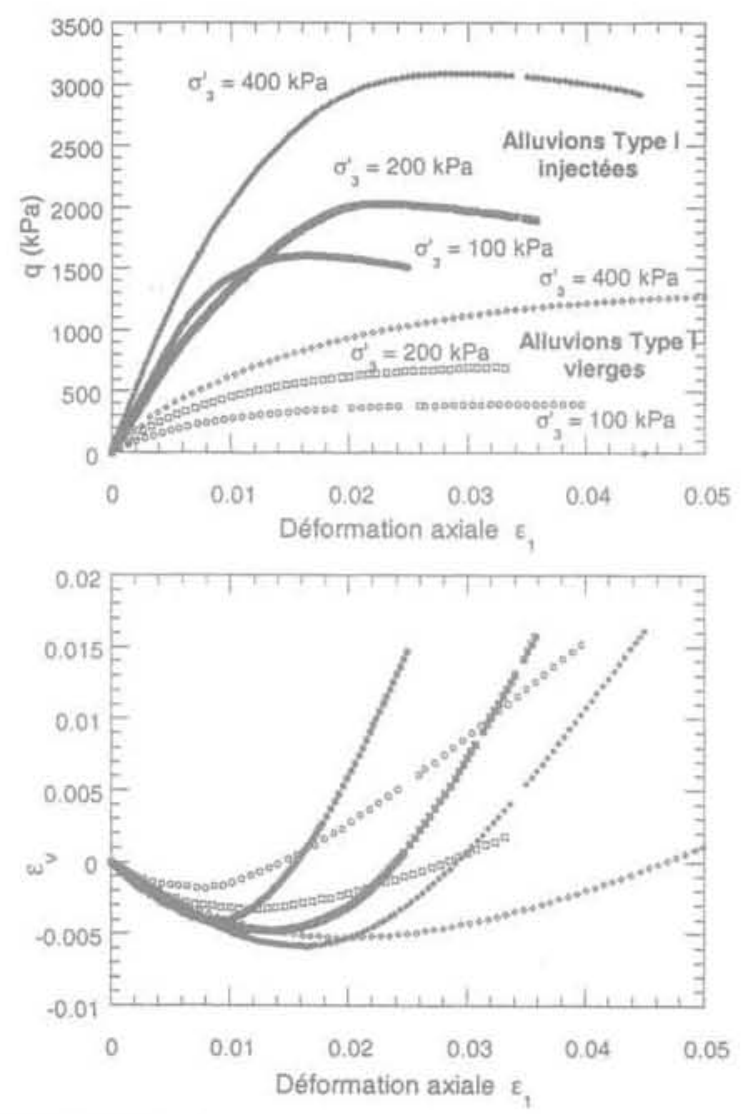

FG. 15 Comparaison du comportement sur les alluvions anciennes de Type $I$ avant et après injection.

Comparison of the behaviour of Type I alluvial deposits before and after grout injection.

de tout traitement. Cet angle diminue également avec la contrainte appliquée. De plus, alors que le taux de dilatance maximal coincide avec le déviateur maximal pour un sol vierge, on s'aperçoit que, pour le sol injecté, le comportement volumique est principalement contractant jusqu'au déviateur maximal, la dilatance n'apparaissant qu'une fois ce déviateur maximal atteint. L'origine de ce comportement dilatant est probablement due à un mécanisme de dégradation progressive de la cimentation (cornme semble le prouver le mode de rupture par des fissures verticales) plutôt qu'à des déplacements des grains les uns par rapport aux autres, par glissements et rotations.

\section{Interprétation conventionnelle}

En reportant, dans le plan ( $\left.\mathrm{p}^{\prime}, \mathrm{q}\right)$, les chemins de contraintes suivis au cours des différents essais, on constate que les points figuratifs de la résistance maximale s'alignent suivant une droite de pente $M_{p}$ supérieure ou égale à la pente obtenue sur le sol vierge. Les valeurs des pentes $\mathrm{M}_{\text {r }}$ sont données dans le Tableau III. Des augmentations de l'angle de frottement interne de l'ordre de 1 à 2 degrés sont ainsi enregistrées pour les éprouvettes de sable de Fontainebleau (Tableau III). Ceci corrobore les observations expérimentales publiées dans la littérature qui indiquent que l'angle de frottement interne n'est que peu modifié par l'imprégnation du squelette granulaire par un coulis (Cambefort, 1967 ; Biarez et al., 1998; Tailliez, 1998). Dans le cas des alluvions anciennes, l'augmentation de l'angle de frottement interne atteint des valeurs de l'ordre de 7 à 9 degrés, quí s'expliquent en grande partie par la densité plus importante des alluvions mises en place dans la colonne.

Les enveloppes de résistance maximale étant approximativement parallèles pour des échantillons de sol vierge et de sol injecté, mis en place à un même indice de densité relative, l'injection de coulis se traduit principalement par l'apparition d'une cohésion. La valeur de la cohésion dépend de (Tableau III) :

- la nature du sol : les essais réalisés ne permettent toutefois pas de proposer une loi d'évolution générale: - de son état de saturation initial : une saturation du sol, préalablement à l'injection, engendre une diminution de la cohésion, toutes choses égales par ailleurs, de l'ordre de $20 \%$. La saturation du sol engendre une dilution du coulis qui favorise son écoulement dans les pores et réduit la concentration de ciment dans un volume du massif de sol:

- de la teneur en ciment du coulis : une augmentation substantielle du rapport C/E, de 0,172 à 0,235 , si elle génère des pressions d'injection plus élevées qui peuvent être préjudiciables aux ouvrages existants, engendre également un accroissement de la cohésion de $44 \%$.

Les valeurs du coefficient de Poisson sont comprises en général entre 0,2 et 0,3 . Les valeurs du module sécant à l'origine de la courbe effort/déforma-

tadieau IIII Paramètres des sols après injection.

Parameters of the elastic perfectly plastic model after injection.

\begin{tabular}{|c|c|c|c|c|c|c|c|}
\hline $\mathrm{E}_{\mathrm{eec}}(\mathrm{MPa})$ & $\begin{array}{c}\sigma^{\prime}=100 \\
\mathrm{kPa}\end{array}$ & $\begin{array}{c}\sigma^{\prime}=200 \\
\mathrm{kPa}\end{array}$ & $\begin{array}{c}\sigma_{\mathrm{TPa}}=400 \\
\mathrm{kPa}\end{array}$ & $v$ & $\begin{array}{c}\sigma_{\mathrm{y}}=100 \\
\mathrm{kPa}\end{array}$ & $\begin{array}{c}\sigma=200 \\
\mathrm{kPa}\end{array}$ & $\begin{array}{c}\sigma_{3}=400 \\
\mathrm{kPa}\end{array}$ \\
\hline $\mathrm{SF}+\mathrm{C} / \mathrm{E}=0,172$ & 533 & 259 & 344 & $\mathrm{SF}+\mathrm{C} / \mathrm{E}=0.172$ & 0,19 & 0,24 & 0,29 \\
\hline $\mathrm{SF}+\mathrm{C} / \mathrm{E}=0,235$ & 346 & 418 & 373 & $\mathrm{SF}+\mathrm{C} / \mathrm{E}=0,235$ & 0,21 & 0,29 & 0,33 \\
\hline $\mathrm{SF}+$ eaU $+\mathrm{CJE}=0,172$ & 294 & 274 & 352 & $\mathrm{SF}=e a u=\mathrm{C} / \mathrm{E}=0,172$ & 0,14 & 0,26 & 0,25 \\
\hline $\mathrm{AATI}+\mathrm{C} / \mathrm{E}=0.172$ & 208 & 151 & 256 & $\mathrm{AATI}+\mathrm{C} / \mathrm{E}=0,172$ & 0,10 & 0,20 & 0.23 \\
\hline $\mathrm{AATII}+\mathrm{C} / \mathrm{E}=0,172$ & 206 & 213 & 279 & $\mathrm{AATH}+\mathrm{C} / \mathrm{E}=0,172$ & 0,21 & 0,23 & 0,19 \\
\hline$\psi(1)$ & $\begin{array}{c}\sigma=100 \\
\mathrm{kPa}\end{array}$ & $\begin{array}{c}\sigma_{\mathrm{A}}=200 \\
\mathrm{kPa}\end{array}$ & $\begin{array}{c}\sigma_{\mathrm{s}}=400 \\
\mathrm{kPa}\end{array}$ & & $\mathrm{M}_{p}$ & $\varphi(\theta)$ & $\mathrm{c}^{\prime}(\mathrm{kPa})$ \\
\hline $\mathrm{SF}+\mathrm{C} / \mathrm{E}=0,172$ & 28,5 & 23,8 & 20,8 & $\mathrm{SF}+\mathrm{C} / \mathrm{E}=0,172$ & 1,67 & 40,7 & 341 \\
\hline $\mathrm{SF}+\mathrm{C} / \mathrm{E}=0,235$ & - & 24,0 & 22,3 & $\mathrm{SF}+\mathrm{C} / \mathrm{E}=0,235$ & 1,70 & 41,5 & 501 \\
\hline $\mathrm{SF}+$ eau $+\mathrm{C} / \mathrm{E}=0,172$ & 20,5 & 20,5 & 19.7 & $\mathrm{SF}+$ eau $+\mathrm{C} / \mathrm{E}=0,172$ & 1,68 & 41,0 & 276 \\
\hline $\mathrm{AAT}+\mathrm{C} / \mathrm{E}=0,172$ & 28.9 & 24,9 & 20,8 & $\mathrm{AATI}+\mathrm{C} / \mathrm{E}=0,172$ & 1,88 & 45,6 & 218 \\
\hline $\mathrm{AATII}+\mathrm{C} / \mathrm{E}=0,172$ & 30,4 & 29,1 & 21,4 & $\mathrm{AATTl}+\mathrm{C} / \mathrm{E}=0.172$ & 1,84 & 44,7 & 313 \\
\hline
\end{tabular}


tion, déterminées pour une déformation de l'ordre de $10^{-3}$, sont considérablement accrues mais ne semblent pas être affectées par la contrainte latérale. Ceci laisse penser que le comportement des sols injectés, dans le domaine des faibles contraintes de cisaillement, est contrôlé par le coulis. Au contraire, la dépendance du déviateur maximal avec la contrainte appliquée montre que, dans le domaine des grandes déformations, le rôle du squelette granulaire, par le mécanisme de frottement, ne peut être négligé.

\section{5}

\section{Facteurs d'amélioration}

A partir des essais triaxiaux présentẻs précédemment, il devient possible de définir les facteurs d'amélioration des paramètres du modèle élastique plastique parfait. Les facteurs d'amélioration sont définis comme le rapport $\mathrm{R}^{*}$ :

$$
R^{*}=\frac{R(\text { injecté })}{R(\text { vierge })}
$$

de la valeur du paramètre $\mathrm{R}$ pour le sol injecté sur la valeur du même paramètre pour le sol vierge de tout traitement (Tableau IV).

On observe expérimentalement que le coefficient de Poisson des sols injectés ne diffère pas des valeurs couramment considérées en mécanique des sols. Des valeurs entre 0,2 et 0,3 sont tout à fait pertinentes à la fois pour le matériau vierge de tout traitement et pour le matériau injecté.

Par ailleurs, les lois d'évolution du module sécant $E_{\text {sec }}\left(\varepsilon=10^{-3}\right)$ (Tableau IV) en fonction de la contrainte appliquée étant différentes pour un sol vierge (loi approximativement linéaire) et pour un sol injecté (quasi-indépendance du module en fonction de la contrainte), le facteur d'amélioration sur le module sécant $\mathrm{E}_{\text {stc }}$ " diminue avec la contrainte appliquée. Des valeurs comprises entre 2 (aux fortes contraintes) et 8 (aux faibles contraintes) sont déduites des essais réalisés. Ceci signifie également que l'effet de l'injection se fait de moins en moins sentir pour des profondeurs de plus en plus grandes. Le facteur d'amélioration dépend également de la nature du traitement d'injection et de la teneur en liant du coulis. Les facteurs d'armélioration donnés ci-dessus sont valables pour un traitement de consolidation des sols pour lequel du coulis à teneur en liant conséquente est injecté. Au contraire, les traitements visant à réduire la perméabilité d'un massif se font souvent en injectant un coulis fortement dosé en bentonite et plus faiblement dosé en ciment. On retiendra donc, pour un traitement de consolidation, qu'un facteur d'amélioration minimal de 1,5 peut être considéré.

Cependant, en raison de la non-linéarité du comportement des sols vierges ou injectés et des erreurs liées à la méthode de mesure de la déformation axiale (erreur de planéité, de verticalité, déformabilité propre de la cellule), le module sécant $\mathrm{E}_{\text {cos }}\left(\varepsilon=10^{-3}\right)$ sous-estime le véritable module élastique, le module d'Young. Des mesures, par propagation d'ondes de compression longitudinales, effectuées à la pression atmosphérique, donnent des valeurs du module d'Young de l'ordre de quelques gigapascals. Le facteur d'amélioration correspondant à de telles valeurs du module d'Young, dans le domaine des très petites déformations, serait donc
TABLEAU IV Facteurs d'amélioration. Improvement ratios.

\begin{tabular}{|c|c|c|c|}
\hline Déviateur $q_{\max }{ }^{*}$ & $\begin{array}{c}\sigma_{\mathrm{a}}^{\prime}=100 \\
\mathrm{kPa}\end{array}$ & $\begin{array}{c}\sigma_{1}^{\prime}=200 \\
\mathrm{kPa}\end{array}$ & $\begin{array}{c}\sigma_{1}=400 \\
\mathrm{kPa}\end{array}$ \\
\hline $\mathrm{SF}+\mathrm{C} / \mathrm{E}=0,172$ & 5,5 & 3,1 & 2,2 \\
\hline $\mathrm{SF}+\mathrm{C} / \mathrm{E}=0,235$ & 6.8 & 4,3 & 2,6 \\
\hline AA Type $I+C / E=0,172$ & 4,1 & 2,9 & 2,4 \\
\hline AA Type II $+\mathrm{C} / \mathrm{E}=0.172$ & 5,5 & - & 2.6 \\
\hline Module sécant E & $\begin{array}{c}\sigma_{2}=100 \\
\mathrm{kPa}\end{array}$ & $\begin{array}{c}\sigma_{j}^{\prime}=200 \\
k P a\end{array}$ & $\begin{array}{c}\sigma_{2}^{\prime}=400 \\
\mathrm{kPa}\end{array}$ \\
\hline $\mathrm{SF}+\mathrm{C} / \mathrm{E}=0,172$ & 8,3 & 2,0 & 1,5 \\
\hline $\mathrm{SF}+\mathrm{C} / \mathrm{E}=0,235$ & 5,4 & 3,2 & 1.7 \\
\hline AA Type $I+C / E=0,172$ & 4,5 & 2,1 & 2,0 \\
\hline \multirow[t]{2}{*}{ AA Type $I I+C / E=0,172$} & 4,4 & 3,1 & 2.5 \\
\hline & $M_{0}+$ & $\Delta \varphi^{\prime}$ & $\Delta c^{\prime}$ \\
\hline $\mathrm{SF}+\mathrm{C} / \mathrm{E}=0,172$ & 1,04 & $+1.6^{\circ}$ & $+341 \mathrm{kPa}$ \\
\hline $\mathrm{SF}+\mathrm{C} / \mathrm{E}=0,235$ & 1,06 & $+2,4^{\circ}$ & $+501 \mathrm{kPa}$ \\
\hline AA Type $I+C / E=0,172$ & 1,26 & $+9,0^{\circ}$ & $+218 \mathrm{kPa}$ \\
\hline AA Type $\Pi+C / E=0,172$ & 1,19 & $+6,9^{\circ}$ & $+313 \mathrm{kPa}$ \\
\hline
\end{tabular}

au moins de 10. Des développements expérimentaux, par suivi du module de cisaillement au cours d'un essai triaxial au moyen de bender elements par exemple, sont nécessaires pour confirmer d'une part le mécanisme de déformation conduisant à la rupture et d'autre part pour avoir une idée plus précise sur le facteur d'amélioration du module en fonction du niveau de déformation considéré

On peut admettre qu'un traitement par imprégnation de coulis ne modifie que peu l'angle de frottement interne d'un sol. L'injection se traduit principalement par l'apparition d'une cohésion qui peut se déduire soit des essais triaxiaux, soit des relations (9) et (11).

$$
c^{\prime} \frac{1-\sin \varphi^{\prime}}{2 \cos \varphi^{\prime}} \times R_{c s i}
$$

Dans le premier cas, les essais effectués montrent une dépendance de la cohésion vis-à-vis de la nature du sol, de son état de saturation, et surtout de la teneur en ciment du coulis. Dans le second cas, si l'on admet que l'angle de frottement interne est maintenu constant au cours de l'injection, la valeur de la cohésion peut se déduire des essais de résistance en compression simple sur le sol injecté et des essais triaxiaux sur le sol vierge de tout traitement. Une marge de sécurité est toutefois considérée : la cohésion introduite dans les calculs d'ouvrages est réduite d'un facteur de sécurité 3 de telle sorte que, pour un sol ayant une cohésion initiale $c_{\text {ini }}^{\prime}$ et pour lequel les essais donneraient une cohésion moyenne $c_{\text {fin }}^{\prime}$ après traitement, la cohésion de calcul $c_{\text {cal }}^{\prime}$ vaut $\left[c^{\prime}{ }_{\text {ni }}+\left(c_{\text {fin }}^{\prime}-c_{\text {ini }}^{\prime} / 3\right)\right]$ à court terme. Un facteur réducteur de 6 peut être considéré à long terme.

Enfin, on s'abstiendra de définir un facteur d'amélioration sur l'angle de dilatance puisque les mécanismes de déformation responsables de la dilatance sont différents. 


\section{Conclusion}

Cette étude démontre le bien-fondê d'une prise en compte systématique, dans les calculs d'ouvrages, de l'amélioration des propriétés mécaniques des sols par une injection de coulis à base de ciment ultrafin. D'une part, cette prise en compte de l'augmentation de la rigidité et de la résistance des sols injectés, moyennant la considération de facteurs de sécurité, peut se traduire par des économies substantielles sur les ouvrages de soutènement à court terme (entre un à six mois, durée moyenne des travaux d'excavation par plot d'injection), mais également à long terme, puisque la plupart des coulis de nouvelle génération ont un effet pérenne. D'autre part, les injections de coulis permettent de diminuer les risques sur le bâti existant par une réduction de l'amplitude des déformations induites dans le sol lors de la construction des ouvrages.

Nous proposons des valeurs des facteurs d'amélioration des paramètres du modèle élastique plastique parfait. Ces valeurs ne s'appliquent, dans un premier temps, qu'au comportement à court terme, notre connaissance plus restreinte du comportement à long terme nous obligeant à une certaine prudence. Dans ces conditions, on peut considérer les facteurs d'amélioration suivants pour un sable injecté à l'aide d'un coulis à base de ciment ultrafin parfaitement mis en œuvre :

- pas de prise en compte d'amélioration pour l'angle de frottement interne;
- amélioration de la cohésion c' fonction de la teneur en liant du coulis et du sol. La cohésion est réduite d'un facteur de sécurité 3 à court terme et de 6 à long terme; - module d'Young E : facteur d'amélioration de 1,5 pour le court terme et 1 pour le long terme.

Ces propositions ne résultent bien évidemment que de l'analyse conservatrice des conclusions de cette étude au travers de l'expérience acquise par la RATP sur les chantiers d'injection. Celle-ci doit être confortée et étendue à d'autres sites et à d'autres matériaux. En particulier, le facteur d'amélioration sur le module tangent à l'origine des courbes effort-déformation sous-estime probablement la véritable amélioration du module d'Young et du module de cisaillement dans le domaine des très petites déformations. Les perspectives de cette étude visent donc essentiellement à proposer des facteurs d'amélioration des propriétés élastiques des sols dans le domaine des petites et très petites déformations.

\section{Textes normatifs}

Norme française NF P 94-059 - Détermination des masses volumiques minimale et maximale des sols non cohérents, 1992.

Norme française NF P 94-070. Essais à l'appareil triaxial de révolution - Généralités, définitions, 1994.

Norme française NF P 94-074 - Essais à l'appareil triaxial - Appareillage - Préparation des éprouvettes - Essais $U U-C U+u-C D$, 1994.

\section{Bibliographie}

Airev D.W. - « Triaxial testing of naturally cemented carbonate soil 1. Journal of Geotechnical Engineering, ASCE, vol.119, n'9, 1993, p. 1379-1399.

Arenzana L.. Krizek R.J.. Pepper S.F. injection of dilute microfine cement suspensions into fine sands x. Proceedings of the 12th International Conference on Soil Mechanics and Foundation Engineering, vol. 2, 1989, p. 1331-1334.

Baiq S. Picornell M. Nazarian S. - u Low strain shear moduli of cemented sands $m$. Journal of Geotechnical and Geoenvironmental Engineering. ASCE, vol. 123, n6, 1997, p. 540-545.

Bennabi A., Levacher D. - « Application d'un produit polymère à la consolidation des sables carbonatés 1 . Revue française de géotechnigue, $n^{\circ}$ 72, 1995 , p. 55-66.

Biarez J., David H., Gouvenot D. Hicher P.Y. Tailliez S., Varjabédian M. - u Comportement mécanique des sols granulaires traités par injection $»$. Revue française de génie civil, vol. 2, n*2, 1998 . p. 255-295.

Cambefort H. - Injection des sols. Tome 1 : Principes et méthodes. Paris, Eyrolles. 1967.

Clough G.W., Sitar N., Bachus R.C. Shafii Rad N. - a Cemented sands under static loading $x$. Journal of the Geotechnical
Engineering Division, ASCE. vol. 107, nGT6, 1981, p. 799-817.

Dano $C$ - - Comportement mécanique des sols injectés. Thèse de Doctorat, Ecole Centrale de Nantes \& Université de Nantes, 2001.

Dupas J.M. Pecker A. - u Static and dynamic properties of sand-cement 3 . Journal of the Geotechnical Engineering Division, ASCE, vol. 105, n' GT3, 1979 , p. $419-436$.

Huang J.T., Airey D.W. - « Properties of artificially cemented carbonate sand $n$. Journal of Geotechnical and Geoenvironmental Engineering, ASCE, vol. 124, n6, 1998, p. 492-499.

Ismail M.A. Joer H.A., Randolph M.F."Sample preparation technique for artificially cemented soils », Geotechnical Testing Journal, vol. 23, $n^{\circ} 2,2000$. p. 171-177.

Kaga M., Yonekura R. - $\alpha$ Estimation of strength of silicate-grouted sand 1 . Soils and Foundations, vol. 31, n 3, 1991. p. 43-59.

Kim M.S. - Étude expérimentale du comportement mécanique des matériaux granulaires sous fortes contraintes. Thèse de doctorat, École centrale de Paris, 1995.

Perret S., Khayat K.H., Ballivy G. - - The effect of degree of saturation of sand on groutability - experimental simulation $\mathrm{n}$. Ground improvement. vol. 4, n 4, 2000 , p. $13-22$

Saxena S.K.. Lastrico R.M. - a Static properties of lightly cemented sand in. Journal of Geotechnical Engineering Division. ASCE, vol. 104, n GT12, 1978, p. 1449 . 1464.

Schwarz L.G. Krizek R.J. - $\alpha$ Effect of preparation technique on permeability and strength of cement-grouted sand $n$. Geotechnical Testing Journal, vol. 17, $n^{\circ}$ 4. 1994a, p. 434-443.

Schwarz L.G., Krizek R.J. - $\approx$ Properties of cement-grouted sand with distance from injection 1. Proceedings of the 13th International Conference on Soil Mechanics and Foundation. Engineering, vol. 1. 1944b, p. 287-290.

Selosse C. - " Météor : une nouvelle ligne de métro entièrement automatique $x$. Tunnels et Ouvrages souterrains, $n^{\circ} 113$, 1992, p. 261-270.

Tailliez S. - Etude expérimentale du comportement mécanique des sols granulaires injectés. Thèse de doctorat, École centrale de Paris, 1998.

Zebovitz S., Krizek R.J. Atmatzidis D.K. a Injection of fine sands with very fine cement grout v. Journal of Geotechnical Engineering, vol. 115, $\mathrm{n}^{2} 12,1989$, p. 1717-1733. 